J. DIFFERENTIAL GEOMETRY

80 (2008) 23-43

\title{
SUBRIEMANNIAN GEOMETRY, A VARIATIONAL APPROACH
}

\author{
Ovidiu Calin \& Der-Chen Chang
}

\begin{abstract}
The paper deals with a variational approach of the subRiemannian geometry from the point of view of Hamilton-Jacobi and Hamiltonian formalism. We present a discussion of geodesics from the point of view of both formalisms, and prove that the normal geodesics are locally length-minimizing horizontal curves.
\end{abstract}

\section{Introduction}

SubRiemannain geometry is considered to be a relatively new branch of geometry, which starts with the study of the Heisenberg group done by Gaveau and Hulanicki in the 1970s ([21], [25]) and Korányi in the 1980s ([26], [27], [28]). However, the first step towards SubRiemannian geometry was made long before, when the principles of Thermodynamics were being discovered.

The study of engines using two isothermes and two adiabatic processes was done by Carnot in 1824 . The study of adiabatic processes continued with the work of Jule. Clausius formulated the second principle of Thermodynamics using the notion of entropy in 1854. Using this background work, Carathèodory stated in his 1909 paper [19] the relationship between the connectivity of two states by adiabatic processes and the integrability of the distribution defined by the kernel of the one-form of work.

Using a supplementary hypothesis on the distribution, Chow proved in 1939 a global connectivity theorem by horizontal curves, where the horizontal curves are the analog of the adiabatic processes in Thermodynamics (see $[\mathbf{2 0}]$ ). The same bracket generating hypothesis was used by Hörmander to show the hypoellipticity of a sum of squares operator (see $[\mathbf{2 4}]$ ).

After Gaveau started the work on Heisenberg group in 1977, the work of Greiner and Beals dealt with properties of the fundamental solution for the sub-Laplacian, and the $\bar{\partial}$-Neumann operator (see [1]). In the mid-1990s they characterized the fundamental solution for the

Received 03/12/2007. 
associated heat operator using the geometry of the Heisenberg group (see $[\mathbf{2}],[\mathbf{3}],[\mathbf{4}])$.

Since then, a series of publications has clarified how subRiemannian geometry can help with the understanding of sub-elliptic operators, not only in the step two case but also in superior step cases (see [11], [12], $[\mathbf{1 3}],[\mathbf{9}],[\mathbf{1 4}],[\mathbf{1 5}],[\mathbf{1 0}],[\mathbf{1 7}])$. Other publications are dedicated only to the properties of subRiemannian manifolds (see $[\mathbf{5}],[\mathbf{6}],[\mathbf{1 8}],[\mathbf{8}],[\mathbf{1 6}]$, $[22],[31])$.

The usual procedure in dealing with this kind of problem is using the Hamiltonian formalism. One of the breakthroughs in the field is the paper of Stricharts [32]. However, there are some unclarified questions in the field about different types of geodesics which are defined by Hamilton-Jacobi formalism, Hamiltonian formalism, and Lagrangian formalism. In the Riemannian geometry all are equivalent (see Calin and Chang [7]). In the subRiemannian case this is not necessarily true. The goal of this paper is to deal with both Hamiltonian and Hamilton-Jacobi formalisms and to state the relationship between them.

The paper is organized in two parts.

The first part of the paper deals with the non-linear Hamilton-Jacobi equation. This is a powerful technique of finding length-minimizing horizontal curves between two given points. We shall show how we can associate with each action a length-minimizing horizontal curve and compute the length of such curve.

In the second part we shall introduce the Hamiltonian function and its properties and describe the subRiemannian geodesics from the Hamiltonian point of view. We will show that the geodesics defined by the Hamiltonian formalism are always locally length minimizing horizontal curves.

The first author was supported by the NSF Grant \#0631541 and the second by a grant from United States Army Research Office and a competitive research grant from Georgetown University.

\section{Preliminary notions}

Let $M$ be a connected differentiable manifold. Since our study is local we may assume that $M=\mathbb{R}^{n}$. We consider the vector fields $X_{1}, X_{2}, \ldots, X_{k}$ on $M$, which are linearly independent and generate the distribution $\mathcal{D}$, called the horizontal distribution. The distribution $\mathcal{D}$ is said to be bracket generating if the vector fields $X_{j}$ together with their iterated Lie brackets

$$
\left[X_{j_{1}}, X_{j_{2}}\right],\left[X_{j_{1}},\left[X_{j_{2}}, X_{j_{3}}\right]\right], \ldots,\left[X_{j_{1}},\left[X_{j_{2}}, X_{j_{3}}\right], \ldots X_{j_{r}}\right]
$$


generate the tangent space $T_{p} M$ at each point $p \in M$. If $f: M \rightarrow \mathbb{R}$ is a smooth function, we define the horizontal gradient of $f$ by

$$
\nabla_{h} f=\sum_{j=1}^{k} X_{j}(f) X_{j} .
$$

We note that $\nabla_{h} f \in \mathcal{D}$.

The energy associated with the function $f$ is defined by

$$
H(\nabla f)=\frac{1}{2}\left|\nabla_{h} f\right|_{g}^{2}=\frac{1}{2} \sum_{j=1}^{k}\left(X_{j}(f)\right)^{2},
$$

where $g$ is the subRiemannian metric, i.e., a metric defined on $\mathcal{D}$ such that the vector fields $X_{j}$ are orthonormal with respect to $g$. It is worth noting that in the case when $\mathcal{D}$ is bracket generating, if $H(\nabla f)=0$, then $f=$ constant.

Any vector field $U$, which belongs to $\mathcal{D}$ is called a horizontal vector field. We note that

$$
U(f)=\sum U^{i} X_{i}(f)=g\left(\sum X_{i}(f) X_{i}, \sum U^{j} X_{j}\right)=g\left(\nabla_{h} f, U\right) .
$$

A curve $\phi:(a, b) \rightarrow M$ is called horizontal if the velocity vector field $\dot{\phi}$ belongs to the horizontal distribution, i.e., $\dot{\phi} \in \mathcal{D}_{\phi}$. If $\mathcal{D}$ is a bracket generating distribution, Chow's theorem [20] states that any two given points can be joined by a piece-wise horizontal curve.

Let $r=n-k$. Consider $r$ linearly independent one-forms $\theta_{1}, \theta_{2}, \ldots, \theta_{r}$, which vanish on the distribution $\mathcal{D}$, i.e., $\theta_{j}\left(X_{\alpha}\right)=0, \forall j=1, \ldots, r$ and $\alpha=1, \ldots, k$. A curve $\phi(s)$ is horizontal if and only if the following constraints on the velocity hold:

$$
\theta_{j}(\dot{\phi}(s))=0 .
$$

Since the distribution $\mathcal{D}$ is bracket generating, it is also non-integrable. The conditions (2.2) are called non-holonomic.

In the following we shall briefly explain the Lagrange-Charpit method of solving a non-linear equation given by

$$
F(x, z, \nabla z)=0
$$

where $x=\left(x_{1}, \ldots, x_{n}\right), \nabla z=\left(\partial_{x_{1}} z, \ldots, \partial_{x_{n}} z\right)$ and $F(x, z, p)$ is a differentiable function defined on an open subset of $\mathbb{R}^{n} \times \mathbb{R} \times \mathbb{R}^{n}$. 
Consider the following ODE system, called the Lagrange-Charpit system of characteristics

$$
\begin{aligned}
\dot{x}_{i} & =\frac{\partial F}{\partial p_{i}} \\
\dot{p}_{i} & =-\left(\frac{\partial F}{\partial x_{i}}+p_{i} \frac{\partial F}{\partial z}\right) \\
\dot{z} & =p_{i} \frac{\partial F}{\partial p_{i}}, \quad i \in\{1, \ldots, n\} .
\end{aligned}
$$

Then the solutions of the above system

$$
x_{i}=x_{i}(t), \quad p_{i}=p_{i}(t), \quad z=z(t)
$$

verify the equation

$$
F(x, p, z)=0 .
$$

The main difficulty is to eliminate the parameter $t$ and write the solution explicitly as $z=z(x)$.

\section{Hamilton-Jacobi formalism}

3.1. The action function. The following definition introduces one of the most important equations of calculus of variations.

Definition 3.1. Let $S$ be a real valued function defined on $\mathbb{R} \times M$. The Hamilton-Jacobi equation for the subRiemannian manifold $M$ is

$$
\frac{\partial S}{\partial \tau}+H(\nabla S)=0
$$

with the initial condition $S_{\mid \tau=0}=S_{0}$. The solution $S$ is called an action function.

Since the Hamilton-Jacobi equation (3.4) is non-linear, it does not always have a unique solution. This will be shown in the following example.

Example 3.2. Consider $M=\mathbb{R}^{3}$ and the Heisenberg vector fields

$$
X_{1}=\partial_{x}+2 y \partial_{t}, \quad X_{2}=\partial_{y}-2 x \partial_{t}
$$

and the associated Hamilton-Jacobi equation

$$
\frac{\partial S}{\partial \tau}+\frac{1}{2} X_{1}(S)^{2}+\frac{1}{2} X_{2}(S)^{2}=0
$$

One can verify the following solutions for equation (3.5)

$$
\begin{aligned}
& S_{1}(x, y, t ; \tau)=-\theta\left(x^{2}+y^{2}\right) \tan (2 \theta \tau)+\theta t, \\
& S_{2}(x, y, t ; \tau)=\theta\left(x^{2}+y^{2}\right) \cot (2 \theta \tau)+\theta t, \\
& S_{3}(x, y, t ; \tau)=\frac{K\left(x^{2}+y^{2}\right)}{2(C+K \tau)}
\end{aligned}
$$

where $\theta, K, C$ are constants. 
If $C=0$, the third solution becomes the well-known Euclidean action $S_{E}=\frac{x^{2}+y^{2}}{2 \tau}$. The second solution can be found in Beals, et al. [4]. We also note that the first and the second solutions are related by

$$
S_{1}\left(x, y, t ; \tau \pm \frac{\pi}{4 \theta}\right)=S_{2}(x, y, t ; \tau) .
$$

The first solution can be obtained as in the following. We look for a solution of the form

$$
S(x, y, t ; \tau)=A(x, y) B(\tau)+C(t) .
$$

Since the vector fields $X_{1}, X_{2}$ have rotational symmetry in $(x, y)$-variables, we shall look for a solution with the same property, i.e.,

$$
y \partial_{x} A-x \partial_{y} A=0 .
$$

Substituting in the equation (3.5) and collecting the similar terms yields

$$
B^{\prime}(\tau)+\frac{1}{2} \frac{\left(\partial_{x} A\right)^{2}+\left(\partial_{y} A\right)^{2}}{A(x, y)} B^{2}(\tau)+\frac{2\left(x^{2}+y^{2}\right)}{A(x, y)} C^{\prime}(t)^{2}=0
$$

Choosing $A(x, y)=2\left(x^{2}+y^{2}\right)$, the coefficients of $B^{2}(\tau)$ and $C^{\prime}(t)^{2}$ become constants, and we get

$$
B^{\prime}(\tau)+4 B^{2}(\tau)+C^{\prime}(t)^{2}=0 .
$$

There is a separation constant $\theta$ such that

$$
\begin{aligned}
C^{\prime}(t) & =\theta \\
B^{\prime}(\tau)+4 B^{2}(\tau) & =-\theta^{2} .
\end{aligned}
$$

Integrating yields

$$
\begin{aligned}
C(t) & =\theta t+C(0) \\
B(\tau) & =-\frac{\theta}{2} \tan (2 \theta \tau) .
\end{aligned}
$$

Hence one possible action is

$$
\begin{aligned}
S(x, y, t ; \tau) & =A(x, y) B(\tau)+C(t) \\
& =-\theta\left(x^{2}+y^{2}\right) \tan (2 \theta \tau)+\theta t
\end{aligned}
$$

where we assumed $C(0)=0$.

In order to obtain the third solution we consider an action function independent of $t$, i.e.,

$$
S=A(x, y) B(\tau) .
$$

A similar method yields the desired solution. 
3.2. Length-minimizing horizontal curves. We shall assume the horizontal distribution is bracket generating, so the connectivity property always holds by Chow's theorem.

Let $p, q \in M$ be two distinct points. We are interested in characterizing the horizontal curves $\phi:[0, \tau] \rightarrow M$ with endpoints $\phi(0)=p$, $\phi(\tau)=q$ for which the length

$$
\ell(\phi)=\int_{0}^{\tau}|\dot{\phi}(t)|_{g} d t
$$

is minimum. Here $g$ denotes the subRiemannian metric.

As in the case of Riemannian geometry, we observe that it suffices to minimize the energy

$$
I(\phi)=\int_{0}^{\tau} \frac{1}{2}|\dot{\phi}(t)|_{g}^{2} d t
$$

instead of length $\ell(\phi)$.

Let $S$ be arbitrarily fixed. Then the functionals

$$
\phi \rightarrow I(\phi)=\int_{0}^{\tau} \frac{1}{2}|\dot{\phi}(t)|_{g}^{2} d t \text { and } \phi \rightarrow J(\phi)=\int_{0}^{\tau}\left(\frac{1}{2}|\dot{\phi}(t)|_{g}^{2}-d S\right)
$$

are related by the relation

$$
J(\phi)=I(\phi)-S(\tau, \phi(\tau))+S(0, \phi(0)) .
$$

Since the endpoints $\phi(0)=p$ and $\phi(\tau)=q$ are fixed, $I(\phi)$ and $J(\phi)$ reach the minimum value for the same curve $\phi(t)$. The relationship between their minima is

$$
\min J(\phi)=\min I(\phi)-S(\tau, q)+S(0, p) .
$$

Since this works for any function $S$, we shall choose it such that the integral $J(\phi)$ has a simplified form. First we shall need the following result.

Lemma 3.3. Let $S:[0, \tau] \times M \rightarrow \mathbb{R}$ be a function and $\phi:[0, \tau] \rightarrow M$ be a horizontal curve. Then

$$
d S_{\mid \phi}=\left({\frac{\partial S}{\partial t}{ }_{\mid \phi}}+g\left(\nabla_{h} S, \dot{\phi}\right)\right) d t
$$

Proof. Let $\phi^{j}(s)=x^{j}(\phi(s))$ be the local components of the curve $\phi$ in a local system of coordinates $\left(x^{1}, \ldots, x^{n}\right)$ on $M$. Applying chain rule and the definition of the horizontal gradient yields

$$
\begin{aligned}
{\frac{d S}{d t}{ }_{\mid \phi}}=\frac{\partial S}{\partial t}{ }_{\mid \phi}+\frac{\partial S}{\partial x^{i}} \frac{d \phi^{i}}{d t}=\frac{\partial S}{\partial t}+\left(\sum_{i=1}^{n} \dot{\phi}^{i} \frac{\partial}{\partial x^{i}}\right) S \\
=\frac{\partial S}{\partial t}+\dot{\phi}(S)=\frac{\partial S}{\partial t}+g\left(\nabla_{h} S, \dot{\phi}\right),
\end{aligned}
$$

where we used (2.1). Multiplying by $d t$ yields (3.8).

q.e.d. 
The main result of this section is given below. It deals with the velocity vector field of a horizontal curve which is length-minimizing. A similar result holds on Riemannian manifolds (see Calin and Chang $[7])$.

Theorem 3.4. Given two distinct points $p, q \in M$, consider the energy functional

$$
\phi \rightarrow I(\phi)=\int_{0}^{\tau} \frac{1}{2}|\dot{\phi}(t)|_{g}^{2} d t,
$$

where $\phi:[0, \tau] \rightarrow M$ is a horizontal curve with fixed end points $\phi(0)=p$ and $\phi(\tau)=q$. Let $S:[0, \tau] \times M \rightarrow \mathbb{R}$ be a solution of the HamiltonJacobi equation (3.4). Then $\phi$ is a minimizer of (3.9) if and only if

$$
\dot{\phi}(t)=\nabla_{h} S_{\mid \phi(t)} .
$$

In this case the minimum value of $I(\phi)$ is $S(0, p)-S(\tau, q)$.

Proof. We shall minimize $J(\phi)$ instead of $I(\phi)$. Using Lemma 3.3 the integral $J(\phi)$ becomes

$$
\begin{aligned}
J(\phi) & =\int_{0}^{\tau}\left(\frac{1}{2}|\dot{\phi}(t)|_{g}^{2} d t-d S_{\mid \phi}\right) \\
& =\int_{0}^{\tau}\left(\frac{1}{2}|\dot{\phi}(t)|_{g}^{2}-\frac{\partial S}{\partial t}_{\mid \phi}-g\left(\nabla_{h} S, \dot{\phi}\right)\right) d t \\
& =\frac{1}{2} \int_{0}^{\tau}\left(|\dot{\phi}(t)|_{g}^{2}-2 g\left(\nabla_{h} S, \dot{\phi}(t)\right)-2 \frac{\partial S}{\partial t}_{\mid \phi}\right) d t \\
& =\frac{1}{2} \int_{0}^{\tau}\left(|\dot{\phi}(t)|_{g}^{2}-2 g\left(\nabla_{h} S, \dot{\phi}(t)\right)+\left|\nabla_{h} S\right|_{g}^{2}-\left|\nabla_{h} S\right|_{g}^{2}-2 \frac{\partial S}{\partial t}_{\mid \phi}\right) d t \\
& =\int_{0}^{\tau}\left(\frac{1}{2}\left|\dot{\phi}-\nabla_{h} S\right|_{g}^{2}-\left(\frac{1}{2}\left|\nabla_{h} S\right|_{g}^{2}+\frac{\partial S}{\partial t}\right)\right) d t .
\end{aligned}
$$

Since $S$ is a solution of the Hamilton-Jacobi equation the above identity becomes

$$
J(\phi)=\int_{0}^{\tau} \frac{1}{2}\left|\dot{\phi}-\nabla_{h} S\right|_{g}^{2} d t .
$$

Hence $J(\phi)$ is minimum if and only if $\left|\dot{\phi}-\nabla_{h} S\right|_{g}^{2}=0$, i.e., when $\dot{\phi}(t)=$ $\nabla_{h} S_{\mid \phi(t)}$. Since in this case $\min J(\phi)=0$, relation (3.7) yields

$$
\min I(\phi)=S(\tau, q)-S(0, p),
$$

which ends the proof.

q.e.d.

The above theorem has a very important consequence for subRiemannian geometry. The following corollary provides the length of a length-minimizing curve in terms of the action value at the end points. 
Corollary 3.5. Let $S$ be a solution of the Hamilton-Jacobi equation. Then the horizontal curves $\phi(s)$ given by $\dot{\phi}(s)=\nabla_{h} S_{\mid \phi(s)}$ are locally length minimizers. The length of the length-minimizing curve $\phi(s)$ given above, which joins $p=\phi(0)$ and $q=\phi(\tau)$ is

$$
\ell(\phi)=\sqrt{2 \tau(S(\tau, q)-S(0, p))} .
$$

Proof. Let $\phi$ be a minimizer of $I(\phi)$. Since in this case the length and the energy integrals are related by the relation $I(\phi)=\frac{1}{2} \ell(\phi)^{2}$, we have

$$
\begin{aligned}
\ell(\phi) & =\int_{0}^{\tau}|\dot{\phi}(s)|_{g} d s=\sqrt{2 \tau \int_{0}^{\tau} \frac{1}{2}|\dot{\phi}(s)|_{g}^{2} d s} \\
& =\sqrt{2 \tau \min I(\phi)}=\sqrt{2 \tau(S(\tau, q)-S(0, p))} .
\end{aligned}
$$

q.e.d.

Remark 3.6. The above result does not exclude the existence of other kind of length minimizers, which cannot be written as a horizontal gradient of the action.

Remark 3.7. Since the Hamilton-Jacobi equation might have more than one solution, the length-minimizing horizontal curve joining two given points $p$ and $q$ might not be unique. Different actions might yield different length-minimizing curves.

Definition 3.8. The length of the horizontal minimizing curve between two points $p$ and $q$ is called the Carnot-Carathéodory distance between $p$ and $q$.

In order to find the Carnot-Carathéodory distance between two given points, one needs to solve the Hamilton-Jacobi equation, and then use the Corollary 3.5 to obtain the length. We shall do this for an explicit example in the following section. In general, for more complicated examples this computation might be almost impossible to perform explicitly.

3.3. An Example: The Heisenberg distribution. Consider the vector fields given by Example 3.2. We shall recover the length of the minimizing horizontal curves by performing an explicit integration of the equation $\dot{\phi}(s)=\nabla_{h} S_{\mid \phi(s)}$, where the action $S$ is

$$
S(x, y, t ; s)=\theta t-\theta\left(x^{2}+y^{2}\right) \tan (2 \theta s),
$$

see Example 3.2. Let $\phi(s)=(x(s), y(s), t(s))$ be a horizontal lengthminimizing curve. We shall write the velocity vector $\dot{\phi}$ in two different ways. Since $\phi(s)$ is a horizontal curve, we have

$$
\begin{aligned}
\dot{\phi}(s) & =\dot{x} \partial_{x}+\dot{y} \partial_{y}+\dot{t} \partial_{t}=\dot{x} X_{1}+\dot{y} X_{2}+\underbrace{(\dot{t}+2 \dot{y} x-2 \dot{x} y)}_{=0} \partial_{t} \\
& =\dot{x}(s) X_{1}+\dot{y}(s) X_{2} .
\end{aligned}
$$


On the other hand, a computation shows

$$
\begin{aligned}
\dot{\phi}(s) & =\nabla_{h} S_{\mid \phi(s)}=\left(X_{1} S\right) X_{1}+\left(X_{2} S\right) X_{2} \\
& =\left(\partial_{x} S+2 y \partial_{t} S\right) X_{1}+\left(\partial_{y} S-2 x \partial_{t} S\right) X_{2} \\
& =2 \theta(y(s)-x(s) \tan (2 \theta s)) X_{1}-2 \theta(x(s)+y(s) \tan (2 \theta s)) X_{2} .
\end{aligned}
$$

Since the coefficients of $X_{1}$ and $X_{2}$ in relations (3.11) and (3.10) should be the same, we get the following ODE system

$$
\begin{aligned}
& \dot{x}(s)=2 \theta(y(s)-x(s) \tan (2 \theta s)) \\
& \dot{y}(s)=-2 \theta(x(s)+y(s) \tan (2 \theta s)) .
\end{aligned}
$$

The $t$-component can be obtained from the horizontality condition

$$
\dot{t}(s)=2 x(s) \dot{y}(s)-2 y(s) \dot{x}(s) .
$$

Differentiating the expression of $\dot{x}(s)$ with respect to $s$ yields

$$
\begin{aligned}
\ddot{x} & =2 \theta\left(\dot{y}-\dot{x} \tan (2 \theta s)-2 \theta x\left(1+\tan ^{2}(2 \theta s)\right)\right) \\
& =2 \theta\left(\dot{y}-2 \theta(y-x \tan (2 \theta s)) \tan (2 \theta s)-2 \theta x\left(1+\tan ^{2}(2 \theta s)\right)\right) \\
& =2 \theta(\dot{y}-2 \theta y \tan (2 \theta s)-2 \theta x) \\
& =2 \theta(\dot{y}-2 \theta(x+y \tan (2 \theta s))) \\
& =2 \theta(\dot{y}+\dot{y})=4 \theta \dot{y} .
\end{aligned}
$$

We have obtained $\ddot{x}=4 \theta \dot{y}$. Similarly we can show $\ddot{y}=-4 \theta \dot{x}$. Hence the components of the length-minimizing curve $\phi=(x, y, t)$ satisfy the system

$$
\ddot{x}=4 \theta \dot{y}, \quad \ddot{y}=-4 \theta \dot{x} .
$$

One may show that the solutions of (3.12) are circles.

As a matter of fact, the system (3.12) is the Euler-Lagrange system associated with the Lagrangian

$$
L(x, y, \dot{x}, \dot{y}, \dot{t})=\frac{1}{2}\left(\dot{x}^{2}+\dot{y}^{2}\right)+\theta(\dot{t}+2 x \dot{y}-2 \dot{x} y),
$$

as the reader can easily verify. The Heisenberg group is one of the examples of subRiemannian manifolds where the Hamilton-Jacobi formalism and the Lagrangian formalism provide the same subRiemannian geodesics. We can add that the Hamiltonian formalism provides the same geodesics, as can be inferred from the second part of the paper.

If one considers the action

$$
S=\theta t+\theta\left(x^{2}+y^{2}\right) \cot (2 \theta s),
$$


then after performing a similar integration in $\dot{\phi}=\nabla_{h} S_{\mid \phi(s)}$ yields

$$
\begin{aligned}
& \dot{x}(s)=2 \theta(y(s)+x(s) \cot (2 \theta s)) \\
& \dot{y}(s)=2 \theta(-x(s)+y(s) \cot (2 \theta s)) .
\end{aligned}
$$

Differentiating leads to the same system (3.12), which means that this action induces the same length-minimizing curves.

Since $\dot{\phi}=\dot{x} X_{1}+\dot{y} X_{2}$, using (3.13)-(3.14) the energy at $\phi(s)$ is

$$
\begin{aligned}
E & =\frac{\dot{x}^{2}(s)+\dot{y}^{2}(s)}{2}=\frac{1}{2} 4 \theta^{2}\left(x^{2}(s)+y^{2}(s)\right)(1+\cot (2 \theta s)) \\
& =\frac{2 \theta^{2}\left(x^{2}(s)+y^{2}(s)\right)}{\sin ^{2}(2 \theta s)}=\frac{2 \theta^{2} r^{2}(s)}{\sin ^{2}(2 \theta s)} .
\end{aligned}
$$

Using (3.12) we have

$$
\frac{d}{d s} E=\frac{d}{d s} \frac{\dot{x}(s)^{2}+\dot{y}(s)^{2}}{2}=\dot{x}(s) \ddot{x}(s)+\dot{y}(s) \ddot{y}(s)=0,
$$

i.e., the energy $E$ is constant. If the parameter $s \in[0, \tau]$, it follows that

$$
E=\frac{2 \theta^{2} r^{2}(\tau)}{\sin ^{2}(2 \theta \tau)}
$$

and hence

$$
\min I(\phi)=\int_{0}^{\tau} E d s=E \tau=\frac{2 \theta^{2} r^{2}(\tau) \tau}{\sin ^{2}(2 \theta \tau)} .
$$

The constant $\theta$ depends on the coordinates of the end points $p$ and $q$. We shall choose $p=(0,0,0)$. Then

$$
\min I(\phi)=S(\tau, q)-S(0, p)=\theta t(\tau)+\theta r(\tau)^{2} \cot (2 \theta \tau) .
$$

Equating (3.16) and (3.15) we obtain the following equation for $\theta$

$$
\frac{t(\tau)}{r(\tau)^{2}}=\frac{2 \theta \tau}{\sin ^{2}(2 \theta \tau)}-\cot (2 \theta \tau)
$$

The equation (3.17) has finitely many solutions as long as $r(\tau) \neq 0$, and at least a solution if $t>0$, see Beals at al. [4]. If $r(\tau)=r(0)=0$, then the equation (3.17) has infinitely many solutions. The solutions are given by $\theta_{n}=\frac{n \pi}{2 \tau}, n \in \mathbb{Z}$. This corresponds to infinitely many energy-minimizing curves between the origin and the point $q=(0,0, t)$, $t>0$.

The lengths of the minimizing horizontal curves joining the origin and $q=(0,0, t)$ are given by Corollary 3.5

$$
\ell_{n}=\sqrt{2 \tau(S(\tau)-S(0))}=\sqrt{2 \tau \theta_{n} t}=\sqrt{n \pi t} .
$$

These lengths were obtained for the first time by Gaveau (see [21]). 
3.4. Solving the Hamilton-Jacobi equation. In this section we shall reduce the problem of finding the solutions of the Hamilton-Jacobi equation to the problem of finding the solution of the eiconal equation. The later equation will be approached in Section $\S 4.3$.

Consider the Hamilton-Jacobi equation

$$
\frac{\partial S}{\partial \tau}+\frac{1}{2} \sum_{j=1}^{k}\left(X_{j} S\right)^{2}=0 .
$$

Looking for a solution of the type $S(x, \tau)=A(\tau)+f(x)$ yields

$$
A^{\prime}(\tau)+H(\nabla f)(x)=0 .
$$

There is a separation constant $\theta>0$ such that

$$
\begin{aligned}
A^{\prime}(\tau) & =-\theta \\
H(\nabla f) & =\theta .
\end{aligned}
$$

The action becomes

$$
S(x, \tau)=-\theta \tau+f(x),
$$

where $f(x)$ is a solution of the eiconal equation

$$
\left|\nabla_{h} f\right|_{g}^{2}=2 \theta
$$

\section{Hamiltonian formalism}

The Hamiltonian formalism is another powerful variational technique applied in subRiemannian geometry. It was first applied by Beals, Gaveau, and Greiner [3], [21], and Stricharts (see [32]). In this part we shall describe the geodesics using Hamiltonian formalism.

4.1. The Hamiltonian function. Consider $M=\mathbb{R}^{n}$ and let $\mathcal{D}=$ $\operatorname{span}\left\{X_{1}, \ldots, X_{k}\right\}$ be the horizontal distribution. The associated Hamiltonian is the principal symbol of the sub-elliptic operator

$$
\Delta_{X}=\frac{1}{2} \sum_{j=1}^{k} X_{j}^{2}
$$

and it can be written as

$$
H(x, p)=\frac{1}{2} \sum_{j=1}^{k}\left\langle X_{j}(x), p\right\rangle^{2},
$$

where $\langle$,$\rangle is the usual inner product on \mathbb{R}^{n}$.

In the following we shall write the Hamiltonian in a more useful way. The Hamiltonian (4.21) can be expanded as a quadratic form in the 
components $p_{i}$ as

$$
H(x, p)=\frac{1}{2} \sum_{i, j=1}^{n} h^{i j}(x) p_{i} p_{j}=\frac{1}{2} h(p, p) .
$$

The coefficients $h^{i j}(x)$ are smooth functions of $x$. Then $h^{i j}$ can be regarded as the components of a symmetric 2-contravariant tensor $h$. Since $H(x, p)$ is a sub-elliptic quadratic form, the coefficients $h^{i j}$ do not define a Riemannian metric, as we shall see later. The matrix $h^{i j}(x)$ is everywhere degenerated. We shall prove this after we provide first a few examples.

Example 4.1. Consider the Heisenberg vector fields

$$
X_{1}=\partial_{x_{1}}+2 x_{2} \partial_{t}, \quad X_{2}=\partial_{x_{2}}-2 x_{1} \partial_{t} .
$$

The Hamiltonian function is

$$
\begin{aligned}
H(x, p) & =\frac{1}{2}\left\langle X_{1}(x), p\right\rangle^{2}+\frac{1}{2}\left\langle X_{2}(x), p\right\rangle^{2} \\
& =\frac{1}{2}\left(p_{1}+2 x_{2} p_{3}\right)^{2}+\frac{1}{2}\left(p_{2}-2 x_{1} p_{3}\right)^{2} \\
& =\frac{1}{2} \sum h^{i j}(x) p_{i} p_{j},
\end{aligned}
$$

where

$$
h^{i j}(x)=\left(\begin{array}{ccc}
1 & 0 & 2 x_{2} \\
0 & 1 & -2 x_{1} \\
2 x_{2} & -2 x_{1} & 4|x|^{2}
\end{array}\right) .
$$

We note that $\operatorname{det} h^{i j}(x)=0$ for any $x$.

Example 4.2. If consider the vector fields

$$
X_{1}=\partial_{x_{1}}+A_{1}(x) \partial_{x_{3}}, \quad X_{2}=\partial_{x_{2}}-A_{2}(x) \partial_{x_{3}},
$$

then a similar computation yields the coefficients

$$
h^{i j}(x)=\left(\begin{array}{ccc}
1 & 0 & A_{1}(x) \\
0 & 1 & -A_{2}(x) \\
A_{1}(x) & -A_{2}(x) & A_{1}(x)^{2}+A_{2}(x)^{2}
\end{array}\right),
$$

which form a degenerate matrix everywhere.

Example 4.3. If $X_{1}=\cos x_{3} \partial_{x_{1}}+\sin x_{3} \partial_{x_{2}}, X_{2}=\partial_{x_{3}}$, we have

$$
h^{i j}(x)=\left(\begin{array}{ccc}
\cos ^{2} x_{3} & \sin x_{3} \cos x_{3} & 0 \\
\sin x_{3} \cos x_{3} & \sin ^{2} x_{3} & 0 \\
0 & 0 & 1
\end{array}\right)
$$

with $\operatorname{det} h^{i j}(x)=0$. 
The following result provides a formula for the coefficients $h^{i j}$ in terms of the components of the horizontal vector fields $X_{1}, \ldots, X_{k}$, which span the distribution $\mathcal{D}$. Let

$$
X_{j}=\sum_{i=1}^{n} a_{j}^{i}(x) \partial_{x_{i}}, \quad j=1, \ldots, k
$$

be the representation of $X_{j}$ in local components, with $\operatorname{rank}\left(a_{j}^{i}(x)\right)=k$, $\forall x$.

\section{Proposition 4.1.}

(i) The coefficients of the quadratic form, which define the Hamiltonian $H$ are given by

$$
h^{\alpha \beta}=\sum_{j=1}^{k} a_{j}^{\alpha} a_{j}^{\beta} .
$$

(ii) The matrix $h^{\alpha \beta}(x)$ is degenerate for any $x$.

Proof

(i) The Hamiltonian function can be written as

$$
H(x, p)=\frac{1}{2} \sum_{j=1}^{k}\left\langle X_{j}(x), p\right\rangle^{2}=\frac{1}{2} \sum_{j=1}^{k}\left(\sum_{i=1}^{n} a_{j}^{i}(x) p_{i}\right)^{2} .
$$

Since $H(x, p)=\frac{1}{2} \sum_{i, j} h^{i j} p_{i} p_{j}$, the coefficients are

$$
\begin{aligned}
h^{\alpha \beta} & =\frac{\partial^{2} H}{\partial p_{\alpha} \partial p_{\beta}}=\frac{1}{2} \frac{\partial^{2}}{\partial p_{\alpha} \partial p_{\beta}}\left(\sum_{j=1}^{k}\left(\sum_{i=1}^{n} a_{j}^{i} p_{i}\right)^{2}\right) \\
& =\frac{\partial}{\partial p_{\alpha}}\left(\sum_{j=1}^{k}\left(\sum_{i=1}^{n} a_{j}^{i} p_{i}\right) a_{j}^{\beta}\right)=\sum_{j=1}^{k} a_{j}^{\alpha} a_{j}^{\beta} .
\end{aligned}
$$

(ii) Using the multi-linearity property and the definition of the determinant we have

$$
\begin{aligned}
\operatorname{det} h^{\alpha \beta} & =\operatorname{det}\left(\sum_{j=1}^{k} a_{j}^{\alpha} a_{j}^{\beta}\right) \\
& =\sum_{j=1}^{k} \sum_{\sigma \in S_{n}} \epsilon(\sigma) a_{j}^{1} a_{j}^{\sigma(1)} a_{j}^{2} a_{j}^{\sigma(2)} \ldots a_{j}^{n} a_{j}^{\sigma(n)} \\
& =\sum_{j=1}^{k} \sum_{\sigma \in S_{n}} \epsilon(\sigma)\left(a_{j}^{1} a_{j}^{2} \ldots a_{j}^{n}\right)\left(a_{j}^{\sigma(1)} a_{j}^{\sigma(2)} \ldots a_{j}^{\sigma(n)}\right) \\
& =\sum_{j=1}^{k} \sum_{\sigma \in S_{n}} \epsilon(\sigma)\left(a_{j}^{1} a_{j}^{2} \ldots a_{j}^{n}\right)^{2}
\end{aligned}
$$




$$
=(\underbrace{\sum_{\sigma \in S_{n}} \epsilon(\sigma)}_{=0})\left(\sum_{j=1}^{k}\left(a_{j}^{1} \ldots a_{j}^{n}\right)^{2}\right)=0 .
$$

q.e.d.

The $h^{i j}(x)$ is the analogue of the raised index metric from Riemannian geometry. Since $h^{i j}(x)$ is nowhere invertible, there is no obvious analogue of the lowered index metric.

As a general rule, the formulas of Riemannian geometry that can be extended in terms of raised indices alone are good candidates for valid formulas in subRiemannian geometry.

The above discussion suggests an alternate definition for the subRiemannian metric.

Definition 4.2. Let $\mathcal{D}=\operatorname{span}\left\{X_{1}, \ldots, X_{k}\right\}$ be a differentiable distribution on the manifold $M$. The contravariant subRiemannian metric $h$ is defined as a symmetric, 2-contravariant tensor, degenerate at every point, such that $\frac{1}{2} \sum_{i, j} h^{i j}(x) p_{i} p_{j}$ is the principal symbol of the operator $\Delta_{X}=\frac{1}{2} \sum_{j} X_{j}^{2}$.

4.2. Regular geodesics and their properties. Hamiltonian formalism can be used to define subRiemannian geodesics. A similar definition makes sense in the Riemannian case.

Definition 4.3. A normal geodesic between the points $A$ and $B$ is a solution $x(s)$ of the Hamiltonian system

$$
\begin{aligned}
\dot{x}^{i}(s) & =\frac{\partial H}{\partial p_{i}} \\
\dot{p}_{i}(s) & =-\frac{\partial H}{\partial x^{i}}, \quad i=1, \ldots, n
\end{aligned}
$$

with the boundary conditions $x(0)=A$ and $x(\tau)=B$.

Proposition 4.4. Along the normal geodesics the Hamiltonian is preserved.

Proof. The proof is a consequence of Hamiltonian equations. Let $(x(s), p(s))$ be a solution of the Hamiltonian system. Since the Hamiltonian $H$ does not depend explicitly on $s$ we have

$$
\begin{aligned}
\frac{d}{d s} H(x(s), p(s)) & =\frac{\partial H}{\partial x^{i}} \dot{x}^{i}+\frac{\partial H}{\partial p_{j}} \dot{p}_{j} \\
& =\frac{\partial H}{\partial x^{i}} \frac{\partial H}{\partial p_{j}}-\frac{\partial H}{\partial p_{j}} \frac{\partial H}{\partial x_{j}} \\
& =0,
\end{aligned}
$$

and hence $H(x(s), p(s))$ is constant along the solutions.

q.e.d. 
The equation of normal geodesics can be described by a second order ODE. In order to do this, it is useful to introduce the raised Christoffel symbols (see Günter [23] and Strichartz [32])

$$
\Gamma^{i a b}(x)=\frac{1}{2}\left(\frac{\partial h^{i a}(x)}{\partial x^{r}} h^{r b}(x)+\frac{\partial h^{i b}(x)}{\partial x^{r}} h^{r a}(x)-\frac{\partial h^{a b}(x)}{\partial x^{r}} h^{r i}(x)\right) .
$$

Proposition 4.5. The equation of normal geodesics are given by

$$
\ddot{x}^{i}(s)=\Gamma^{i}(x)(p(s), p(s)), \quad i=1, \ldots, n,
$$

where $(x(s), p(s))$ is a solution of the Hamiltonian system.

Proof. The first Hamiltonian equation can be written as $\dot{x}^{i}(s)=$ $h^{i j}(x(s)) p_{j}$. Differentiating with respect to $s$, and using the second Hamiltonian equation, we obtain

$$
\begin{aligned}
\ddot{x}^{i}(s)= & \frac{d}{d s}\left(h^{i j}(x(s)) p_{j}(s)\right) \\
= & \frac{\partial h^{i j}(x)}{\partial x^{r}} \dot{x}^{r}(s) p_{j}(s)+h^{i j}(x) \dot{p}_{j}(s) \\
= & \frac{\partial h^{i j}(x)}{\partial x^{r}} h^{r l}(x) p_{l} p_{j}-\frac{1}{2} h^{i j}(x) \frac{\partial h^{a b}}{\partial x^{j}} p_{a} p_{b} \\
= & \frac{\partial h^{i b}(x)}{\partial x^{r}} h^{r a}(x) p_{a} p_{b}-\frac{1}{2} h^{i j}(x) \frac{\partial h^{a b}(x)}{\partial x^{j}} p_{a} p_{b} \\
= & \frac{1}{2} \frac{\partial h^{i b}(x)}{\partial x^{r}} h^{r a}(x) p_{a} p_{b}+\frac{1}{2} \frac{\partial h^{i b}(x)}{\partial x^{r}} h^{r a}(x) p_{a} p_{b} \\
& -\frac{1}{2} h^{i j}(x) \frac{\partial h^{a b}(x)}{\partial x^{j}} p_{a} p_{b} \\
= & \frac{1}{2}\left(\frac{\partial h^{i a}}{\partial x^{r}} h^{r b}+\frac{\partial h^{i b}}{\partial x^{r}} h^{r a}-\frac{\partial h^{a b}}{\partial x^{j}} h^{i j}\right) p_{a} p_{b} \\
= & \Gamma^{i a b} p_{a} p_{b}=\Gamma^{i}(p, p) .
\end{aligned}
$$

q.e.d.

The main difficulty in the study of the subRiemannian geodesics is that we cannot usually solve for $p(s)$ in terms of $x(s)$, like in the case of Riemannian geometry. This happens because $\operatorname{det} h^{i j}(x)=0$, so that the equation $\dot{x}^{i}(s)=h^{i j}(x) p_{j}(s)$ cannot be inverted in a usual way to obtain $p_{j}(s)$. However, in some cases this can be done explicitly and we shall present one example below.

Example 4.4. This example deals with the geodesics on the Heisenberg group. Substituting the formulas of $h^{i j}$ given by Example 4.1 into 
the formula for the raised Christoffel symbols (4.24) yields

$$
\begin{array}{lll}
\Gamma^{111}=0 & \Gamma^{211}=0 & \Gamma^{311}=0 \\
\Gamma^{112}=0 & \Gamma^{212}=0 & \Gamma^{312}=0 \\
\Gamma^{113}=0 & \Gamma^{213}=-2 & \Gamma^{313}=4 x^{1} \\
\Gamma^{123}=2 & \Gamma^{223}=0 & \Gamma^{323}=4 x^{2} \\
\Gamma^{133}=-8 x^{1} & \Gamma^{222}=0 & \Gamma^{333}=0 \\
\Gamma^{122}=0 & \Gamma^{233}=-8 x^{2} & \Gamma^{322}=0 .
\end{array}
$$

The other coefficients can be obtained using the symmetry in the last two indices $\Gamma^{i j k}=\Gamma^{i k j}$. The equations of the normal geodesics (4.25) become

$$
\begin{aligned}
& \ddot{x}^{1}(s)=4 p_{3}\left(p_{2}-2 x^{1} p_{3}\right) \\
& \ddot{x}^{2}(s)=-4 p_{3}\left(p_{1}+2 x^{2} p_{3}\right) \\
& \ddot{x}^{3}(s)=8 p_{3}\left(x^{1} p_{1}+x^{2} p_{2}\right) .
\end{aligned}
$$

Since $\dot{p}_{3}=-\frac{\partial H}{\partial x^{3}}=0$ it follows that $p_{3}$ is a first integral of motion. The Hamiltonian equations $\dot{x}^{i}=h^{i j} p_{j}$ become

$$
\begin{aligned}
& \dot{x}^{1}(s)=\frac{\partial H}{\partial p_{1}}=p_{1}+2 x^{2} p_{3} \\
& \dot{x}^{2}(s)=\frac{\partial H}{\partial p_{2}}=p_{2}-2 x^{1} p_{3} .
\end{aligned}
$$

Substituting in (4.26-4.27) yields

$$
\begin{aligned}
& \ddot{x}^{1}(s)=4 p_{3} \dot{x}^{2}(s) \\
& \ddot{x}^{2}(s)=-4 p_{3} \dot{x}^{1}(s),
\end{aligned}
$$

which is the system (3.12) with $\theta=p_{3}, x=x^{1}$ and $y=x^{2}$.

We can solve for the momenta $p_{1}, p_{2}$ in terms of $x^{1}$ and $x^{2}$, keeping $p_{3}$ constant

$$
\begin{aligned}
& p_{1}=\dot{x}^{1}-2 x^{2} p_{3} \\
& p_{2}=\dot{x}^{2}+2 x^{1} p_{3} \\
& p_{3}=\text { constant. }
\end{aligned}
$$

The momentum $p_{3}$ is constant along the solution and depends on the boundary conditions. Given the boundary conditions $x^{i}(0)=x_{0}^{i}$ and $x^{i}(\tau)=x_{f}^{i}$, the constant $p_{3}$ might not be unique (see Gaveau [4] and Section $§ 3.3)$.

Using this, we can find an expression for the component $x^{3}$

$$
\begin{aligned}
\ddot{x}^{3}(s) & =8 p_{3}\left(x^{1} p_{1}+x^{2} p_{2}\right)=8 p_{3}\left(x^{1} \dot{x}_{1}+x^{2} \dot{x}^{2}\right) \\
& =4 p_{3} \frac{d}{d s}\left(x^{1} \dot{x}^{1}+x^{2} \dot{x}^{2}\right),
\end{aligned}
$$


so $\dot{x}^{3}(s)=4 p_{3}\left(\left(x^{1}\right)^{2}+\left(x^{2}\right)^{2}\right)+C$.

The following result is very important since it shows that the solutions of the Hamiltonian system provide curves tangent to the horizontal distribution.

Theorem 4.6. Any normal geodesic is a horizontal curve.

Proof. Let $\theta_{1}, \ldots, \theta_{r}$ be the one-forms which vanish on the horizontal distribution $\mathcal{D}=\operatorname{span}\left\{X_{1}, \ldots, X_{k}\right\}$. Let $\theta_{\alpha}=\theta_{\alpha}^{j} d x_{j}$ and $X_{j}=a_{j}^{i} \partial_{x_{i}}$, where we make the summation convention over the repeated indices. We have

$$
\theta_{\alpha}\left(X_{j}\right)=\theta_{\alpha}^{i} a_{j}^{i}=0, \quad \forall \alpha=1, \ldots r, \quad j=1, \ldots, k .
$$

If $x(s)$ is a normal geodesic, then

$$
\dot{x}^{i}(s)=h^{i j} p_{j} .
$$

Substituting the expression for $h^{i j}$ given by (4.23) in (4.29) yields

$$
\dot{x}^{i}(s)=a_{l}^{i} a_{l}^{j} p_{j} .
$$

Using (4.28) and (4.30) yields

$$
\begin{aligned}
\theta_{\alpha}(\dot{x}(s)) & =\left(\theta_{\alpha}^{\beta} d x_{\beta}\right)\left(\dot{x}^{i}(s) \partial_{x_{i}}\right)=\theta_{\alpha}^{i} \dot{x}^{i}(s) \\
& =\theta_{\alpha}^{i} a_{l}^{i} a_{l}^{j} p_{j}=(\underbrace{\theta_{\alpha}^{i} a_{l}^{i}}_{=0}) a_{l}^{j} p_{j}=0,
\end{aligned}
$$

which shows that the velocity $\dot{x}$ satisfies the non-holonomic constraints induced by the one-forms $\theta_{\alpha}$. Hence $x(s)$ is a horizontal curve. q.e.d.

4.3. The eiconal equation. Let $c>0$ be a constant. We are interested in finding the solutions of the subRiemannian eiconal equation

$$
H(\nabla f)=c .
$$

This equation can be written as

$$
\frac{1}{2} \sum_{j=1}^{k}\left\langle X_{j}(x), \nabla f\right\rangle^{2}=c .
$$

In order to solve the above eiconal equation, we use the LagrangeCharpit method by choosing

$$
\begin{aligned}
F(x, f, p) & =\frac{1}{2} \sum_{j=1}^{k}\left\langle X_{j}(x), p\right\rangle^{2}-c \\
& =H(x, p)-c
\end{aligned}
$$


and writing the system of characteristics

$$
\begin{aligned}
\dot{x}_{i} & =\frac{\partial F}{\partial p_{i}}=\frac{\partial H}{\partial p_{i}} \\
\dot{p}_{i} & =-\frac{\partial F}{\partial x_{i}}=-\frac{\partial H}{\partial x_{i}} \\
\dot{f} & =\sum_{i=1}^{k} p_{i} \frac{\partial F}{\partial p_{i}}=\sum_{i=1}^{k} p_{i} \frac{\partial H}{\partial p_{i}} .
\end{aligned}
$$

Since the first $2 n$ equations form the Hamiltonian system associated with $H(x, p)$, it follows that $x_{i}$ are the components of a normal geodesic. Since the Hamiltonian $H(x, p)$ is homogeneous of degree 2 in the variable $p$, then Euler's formula for homogeneous functions yields

$$
\sum_{i=1}^{k} p_{i} \frac{\partial H}{\partial p_{i}}=2 H=E \text { (the constant of energy). }
$$

The third Lagrange-Charpit equation becomes $\dot{f}(t)=E$. Hence the solution of $(4.31)$ is

$$
f(t)=E t+f(0)
$$

where the constant of energy $E$ depends on the initial and final values of $x(s)$.

4.4. Normal geodesics as length minimizing curves. In this section we shall show the relationship between the normal geodesics and the length minimizers provided by Corollary 3.5.

Proposition 4.7. Let $c(s)=\left(x_{1}(s), \ldots, x_{n}(s)\right)$ be a normal geodesic. Then $\dot{c}(s)=\nabla_{h} S_{\mid c(s)}$, with the action $S$ given by (3.19).

Proof. Let $x(s)$ be a normal geodesic and $(x, p)$ be a solution of the Hamiltonian system. Using $X_{\alpha} S=X_{\alpha} f$ we get $\nabla_{h} S=\nabla_{h} f$, so it suffices to show $\dot{c}=\nabla_{h} f$. Since $f$ satisfies the eiconal equation then $f$ will satisfy the Lagrange Charpit equation (4.32), which can be written as

$$
d f=\sum p_{i} d x_{i}
$$

Hence

$$
p_{i}=\frac{\partial f}{\partial x_{i}}
$$

along the solutions of the Hamiltonian system. Using (4.33) yields

$$
X_{\alpha} f=\sum a_{\alpha}^{i} \frac{\partial f}{\partial x_{i}}=\sum a_{\alpha}^{i} p_{i},
$$


and hence

$$
\begin{aligned}
\dot{x}_{j} & =\frac{\partial H}{\partial p_{j}}=\sum h^{i j}(x) p_{i}=\sum a_{\alpha}^{j} a_{\alpha}^{i} p_{i} \\
& =\sum a_{\alpha}^{j} X_{\alpha} f .
\end{aligned}
$$

The velocity vector field becomes

$$
\begin{aligned}
\dot{c} & =\sum_{j} \dot{x}_{j} \partial_{x_{j}}=\sum_{j, \alpha} a_{\alpha}^{j} X_{\alpha} f \partial_{x_{j}}=\sum_{\alpha}\left(X_{\alpha} f \sum_{j} a_{\alpha}^{j} \partial_{x_{j}}\right) \\
& =\sum_{\alpha}\left(X_{\alpha} f\right) X_{\alpha}=\nabla_{h} f=\nabla_{h} S,
\end{aligned}
$$

which ends the proof.

q.e.d.

Theorem 4.8. The normal geodesics are locally length-minimizing horizontal curves.

Proof. Let $c(s)$ be a normal geodesic. By Theorem $4.6 c(s)$ is a horizontal curve. Since we can write locally $\dot{c}(s)=\nabla_{h} S_{\mid c(s)}$, where $S$ is a solution of Hamilton-Jacobi equation, then Corollary 3.5 yields that $c(s)$ locally minimizes length.

q.e.d.

The reverse statement of the above theorem is not true. The first example of horizontal curves which locally minimizes length but does not satisfy the Hamiltonian system was constructed by Montgomery [30] in 1994 and by Liu and Sussman [29] in 1995. In [29] the following vector fields are considered

$$
X=\partial_{x}, \quad Y=(1-x) \partial_{y}+x^{2} \partial_{t} .
$$

The curve $\gamma:[a, b] \rightarrow \mathbb{R}^{3}, \gamma(s)=(0, s, 0)$ is not a subRiemannian geodesic (does not satisfy the Hamiltonian system) but it minimizes length for $b-a<\frac{2}{3}$.

\section{References}

[1] R. Beals \& P.C. Greiner, Calculus on Heisenberg manifolds, Ann. Math. Studies, 119, Princeton University Press, Princeton, NJ, 1988, MR 0953082, Zbl 0654.58033.

[2] R. Beals, B. Gaveau, \& P.C. Greiner, On a geometric formula for the fundamental solution of subelliptic Laplacians, Math. Nachr. 181 (1996) 81-163, MR 1409074, Zbl 0864.35005.

[3] Complex Hamiltonian mechanics and parametrices for subelliptic Laplacians, I, II, III, Bull. Sci. Math., 21 (1997) 1-36, 97-149, 195-259, MR 1431098, Zbl 0886.35001, MR 1435337, Zbl 0886.35002, MR 1444454, Zbl 0886.35003.

[4] Hamilton-Jacobi theory and the heat kernel on Heisenberg groups, J. Math. Pures Appl. 79(7) (2000) 633-689, MR 1776501, Zbl 0959.35035.

[5] A. Bellaïche, Sub-Riemannian Geometry, Progr. Math., 144, Birkhäuser, Basel, 1996, MR 1421821. 
[6] O. Calin, Geodesics on a certain step 2 sub-Riemannian manifold, Annals of Global Analysis and Geometry 22 (2002) 317-339, MR 1935860, Zbl 1030.53034.

[7] O. Calin \& D.C. Chang, Geometric Mechanics on Riemannian Manifolds, Applications to Partial Differential Equations, Applied and Numerical Analysis, Birkäuser, 2004, MR 2097345, Zbl 1062.58024.

[8] _ Geometric Mechanics on a step 4 subRiemannian manifold, Taiwanese Journal of Mathematics, June 2005, 9(2) 261-280, MR 2142577, Zbl 1088.53021.

[9] , The Geometry on a step 3 Gruhsin model, Arbeitsgruppe Partielle Differentialgeichungen und komplexe Analysis, Universitat Potsdam, ISBN 1437739X, Applicable Analysis 84(2) (2005) 111-129, MR 2114558.

[10] O. Calin, D.C. Chang, \& S. Fricke, Fundamental solutions for a family of subelliptic PDEs, Pure and Applied Mathematics Quarterly 3(2) (2007) 1-23.

[11] O. Calin, D.C. Chang, \& P.C. Greiner, Real and complex Hamiltonian mechanics on some SubRiemannian manifolds, Asian Journal of Mathematics 18(1) (2004) 137-160, MR 2128302, Zbl 1074.53024.

[12] - On a Step 2(k+1) Sub-Riemannian manifold, Journal of Geometric Analysis 14(1) (2004) 1-18, MR 2030572, Zbl 1056.53020.

[13] _ Geometric Analysis on SubRiemannian Manifolds, Advances in Analysis: Proceedings of the 4th ISAAC Congress, World Scientific (ed. by M.W. Wong), 17-38, 2005, MR 2184921, Zbl 1093.53034.

[14] _ Geometric mechanics on the Heisenberg group, Bulletin of the Institute of Mathematics, Academia Sinica 33(3) (Sept. 2005) 185-252, MR 2165285, Zbl 1092.53023.

[15] _ Geometric analysis on the Heisenberg group and its generalizations, AMS/IP Studies in Advanced Mathematics, 40, American Mathematical Society, Providence, RI, International Press, Somerville, MA, 2007, MR 2310372.

[16] O. Calin, D.C. Chang, P. Greiner, \& Y. Kannai, On the geometry induced by a Grusin operator, Proceedings of International Conference on Complex Analysis and Dynamical Systems (ed. by L. Karp and L. Zalcman), Contemporary Math., Amer. Math. Soc. 382 (2005) 89-111, MR 2175879, Zbl 1096.53019.

[17] O. Calin, D.C. Chang, \& Stephen S.T. Yau, Periodic solutions for a family of Euler-Lagrange systems, Asian J. Math. 11(1) (2007) 69-87, MR 2304582.

[18] O. Calin \& V. Mangione, Geodesics with constraints on Heisenberg manifolds, Results in Mathematics 44 (2003) 44-53, MR 2011905, Zbl 1043.37046.

[19] C. Carathéodory, Untersuchungen über die Grundlagen der Thermodynamik (German), Math. Ann. 67(3) (1909) 355-386, MR 1511534.

[20] W.L. Chow, Über Systeme von linearen partiellen Differentialgleichungen erster Ordnung (German), Math. Ann. 117 (1939) 98-105, MR 0001880, Zbl 0022.02304 .

[21] B. Gaveau, Principe de moindre action, propagation de la chaleur et estimes sous elliptiques sur certains groupes nilpotents, Acta Math. 139(1-2) (1977) 95-153, MR 0461589, Zbl 0366.22010.

[22] P. Greiner \& O. Calin, On subriemannian geodesics, Anal. Appl. (Singap.) 1(3) (2003) 289-350, MR 1993340, Zbl 1056.53021.

[23] N.C. Günter, Hamiltonian mechanics and optimal control, Thesis, Harvard University, 1982. 
[24] L. Hörmander, Hypoelliptic second order differential equations, Acta Math. 119 (1967) 147-171, MR 0222474, Zbl 0156.10701.

[25] A. Hulanicki, The distribution of energy in the Brownian motion in the Gaussian field and analytic-hypoellipticity of certain subelliptic operators on the Heisenberg group, Studia Math. 56(2) (1976) 165-173, MR 0418257, Zbl 0336.22007.

[26] A. Koranyi, Geometric aspects of analysis on the Heisenberg group, Topics in modern harmonic analysis, Vol. I, II (Turin/Milan, 1982), 209-258, Ist. Naz. Alta Mat. Francesco Severi, Rome, 1983, MR 0748865, Zbl 0537.22009.

[27] _ Geometric properties of Heisenberg-type groups, Adv. in Math. 56(1) (1985) 28-38, MR 0782541, Zbl 0589.53053.

[28] A. Koranyi \& H.M. Reimann, Quasiconformal mappings on the Heisenberg group, Invent. Math. 80(2) (1985) 309-338, MR 0788413, Zbl 0567.30017.

[29] W. Liu \& J. Sussmann, Shortest paths for sub-Riemannian metrics on ranktwo distributions, Mem. Amer. Math. Soc. 118(564) (1995), MR 1303093, Zbl 0843.53038 .

[30] R. Montgomery, Abnormal minimizers, SIAM J. Control Optim. 32(6) (1994) 1605-1620, MR 1297101, Zbl 0816.49019.

[31] _ A tour of subriemannian geometries, their geodesics and applications, Mathematical Surveys and Monographs, 91, American Mathematical Society, Providence, RI, 2002, MR 1867362, Zbl 1044.53022.

[32] R. Strichartz, Sub-Riemannian geometry, J. Differential Geom. 24(2) (1986) 221-263, MR 0862049, Zbl 0609.53021.

EASTERN Michigan UNIVERSITY YPSILANTI, MI 48197

E-mail address: ocalin@emich.edu DEPARTMENT OF MATHEMATICS

GeORGETOWn University WASHINGTON 20057, DC

E-mail address: chang@georgetown.edu 